\title{
Therapeutic effect and mechanism of 4-phenyl butyric acid on renal ischemia-reperfusion injury in mice
}

\author{
XINLEI WANG, YANG ZHANG, KUN WUYUN and HAIXIA GONG \\ Department of Anesthesiology, The First Affiliated Hospital of Nanchang University, Nanchang, Jiangxi 330006, P.R. China
}

Received December 2, 2020; Accepted August 6, 2021

DOI: $10.3892 / \mathrm{etm} .2021 .11067$

\begin{abstract}
The aim of the present study was to explore the effects and possible mechanism of 4-phenylbutyric acid (4-PBA) on renal ischemia-reperfusion injury (RIRI) in mice. A RIRI model of HK-2 cells was constructed using hypoxia/reoxygenation $(\mathrm{H} / \mathrm{R})$ treatment. Dexmedetomidine and 4-PBA were used to treat the cells before and after modeling. Apoptosis and expression levels of cyclophilin D (CypD), cytochrome $c$, eukaryotic translation initiation factor $2 \alpha(\mathrm{eIF} 2 \alpha)$, glucose-regulated protein 78 (GRP78), intercellular adhesion molecule (ICAM)-1 and vascular adhesion molecule (VCAM)-1 were measured using flow cytometry, western blotting and immunohistochemistry. The renal volume, weight and renal arterial resistance index (RRI) were determined using the renal ischemia model. Compared with untreated model cells, 4-PBA treatment significantly decreased apoptosis and the expression levels of CypD, Cytochrome $c$, eIF $2 \alpha$ and GRP78 in HK-2 cells. There was no significant change in renal volume and weight after modeling, but RRI was significantly decreased after 4-PBA treatments in the model. Western blotting and immunohistochemistry analysis demonstrated that 4-PBA treatment also significantly decreased the expression of ICAM-1 and VCAM-1. Overall, 4-PBA had a therapeutic effect on RIRI in mice. This protection may be mediated by decreasing the expression levels of CypD, Cytochrome $c, \operatorname{eIF} 2 \alpha$ and GRP78, and subsequent reduction of cellular oxygen free radicals and apoptosis, leading to an alleviated endoplasmic reticulum stress response and RIRI.
\end{abstract}

Correspondence to: Professor Haixia Gong, Department of Anesthesiology, The First Affiliated Hospital of Nanchang University, 17 Yongwaizheng Street, Nanchang, Jiangxi 330006, P.R. China

E-mail: ghx1510@163.com

Abbreviations: ICAM-1, intercellular adhesion molecule-1; VCAM-1, vascular adhesion molecule-1; DEX, dexmedetomidine; 4-PBA, 4-phenylbutyric acid; RRI, renal arterial resistance index; RIRI, renal ischemia-reperfusion injury; $H / R$, hypoxia/reoxygenation; ERS, endoplasmic reticulum stress

Key words: dexmedetomidine, 4-phenylbutyric acid, renal ischemia-reperfusion injury, apoptosis, endoplasmic reticulum stress

\section{Introduction}

Renal ischemia-reperfusion injury (RIRI) refers to renal function damage due to failed functional recovery after ischemia-reperfusion during kidney operations such as transplantation and kidney stone surgery. RIRI can sometimes be irreversible (1). Renal ischemia-reperfusion causes numerous pathophysiological changes that results in a poor prognosis. No effective interventions are available to deal with RIRI. Due to highly vascularized tissues, unique vasculature and high oxygen consumption, RIRI can often lead to renal failure and other diseases with high mortality rates $(2,3)$.

The mechanisms underlying RIRI are complex and have not been fully elucidated. At present, it is generally hypothesized that RIRI is associated with $\mathrm{Ca}^{+}$overload, production of oxygen free radicals, activation of cell adhesion molecules, involvement of chemokines and action of white blood cells $(4,5)$. Clinically, no targeted drug is currently available to treat RIRI, although a number of drugs are being used with some curative effect. These include caspase inhibitors, antioxidants and P-selectin antagonists (6).

Dexmedetomidine (DEX) is a highly selective $\alpha 2$-adrenergic agonist; it has anti-sympathetic, sedative and analgesic effects. Studies have demonstrated that DEX can inhibit the release of inflammatory factors and suppress the oxidative stress response to protect organs (7-9). With regards to RIRI, DEX has been revealed to alleviate ischemia-reperfusion-induced RIRI in both animal and human experiments, but its dose-response relationship and underlying mechanism are still unclear (8). Moreover, 4-phenylbutyric acid (4-PBA) is a low molecular weight fatty acid (10). A number of studies have confirmed that 4-PBA can be used as a molecular chaperone to reverse the incorrect displacement or incorrect aggregation of protein molecules to form a normal spatial structure $(11,12)$. This decreases the overload of endoplasmic reticulum stress (ERS), suppresses the signal induction of ERS and alleviates the tissue damage caused by ERS (10). Furthermore, 4-PBA decreases hepatocyte apoptosis in hepatic ischemia-reperfusion injury and alleviates cerebral and spinal cord ischemic injury (13). However, the mechanism by which 4-PBA exerts its therapeutic effect on RIRI is largely unknown.

To the best of our knowledge the present study was the first to use a hypoxia/reoxygenation (H/R) model of the human renal tubular epithelial HK-2 cell line and mice and DEX as positive control to study the therapeutic effect and possible mechanism 
of 4-PBA on RIRI at the cellular and animal levels. The findings could provide evidence to develop improved therapeutic strategies for the disease.

\section{Materials and methods}

Experimental cells and animals. The human renal tubular epithelial HK-2 cell line (SCSP-511) was purchased from The Cell Bank of Type Culture Collection of The Chinese Academy of Sciences and cultured in 10-cm adherent culture dishes containing DMEM with 10\% FBS (Jiangsu KeyGEN BioTECH Co., Ltd.) at $37^{\circ} \mathrm{C}$ in a $5 \% \mathrm{CO}_{2}$ atmosphere. A total of 20 male C57 mice (6-8-weeks; weight, 20-25 g) were obtained from SLEK Lab Animal Center of Shanghai (permit no. Hunan 2019-0004). The animals were kept at a temperature of $20-26^{\circ} \mathrm{C}$, in $40-70 \%$ humidity and a $12 / 12 \mathrm{~h}$ light/dark cycle, with access to filtered water and food ad libitum. Animal studies were approved by The Animal Research Ethics Committee of The First Affiliated Hospital of Nanchang University (approval no. 2019-067). At the end of experiments, mice were euthanized with an overdose of $\mathrm{CO}_{2}$ gas with a $\mathrm{CO}_{2}$ replacement rate of $20 \%$ of the cage volume per min (5 1/min), according to the AVMA Guidelines for Euthanasia (14).

Reagents and instruments. DEX injection (national permit H20130027) was purchased from Chenxin Pharmaceutical Co.,Ltd.4-PBA (cat.no.L15M6D1) was sourced from Shanghai YuanYe Biotechnology Co., Ltd. The Annexin V-FITC/PI Apoptosis kit (cat. no. AP101-100-kit) was purchased from Multisciences (Lianke) Biotech Co., Ltd. Cell lysis buffer (cat. no. C1053) was obtained from Applygen Technologies, Inc. BCA protein quantitative kit (cat. no. CW0014S), neutral resin (cat. no. CW0136) and Diaminobenzidine Substrate kit (cat. no. CW00125) were purchased from CoWin Biosciences. Mouse monoclonal antibody against GAPDH $(1: 2,000$; cat. no. TA-08), goat horseradish peroxidase-conjugated antibody for mouse IgG (H + L; 1:2,000; cat. no. ZB-2305) and goat horseradish peroxidase-conjugated antibody for rabbit IgG (H + L; 1:2,000; cat. no. ZB-2301) were obtained from OriGene Technologies, Inc. Rabbit monoclonal antibody against glucose-regulated protein 78 (GRP78; 1:3,000; cat. no. 11587-1-ap) was purchased from ProteinTech Group, Inc. Rabbit monoclonal antibodies against eukaryotic translation initiation factor $2 \alpha$ (eIF2 $\alpha ; 1: 1,000$; cat. no. AF6087), CypD (1:1,000; cat. no. DF3147), cytochrome $c(1: 1,000$; cat. no. AF0146), intercellular adhesion molecule 1 (ICAM-1; 1:1,000; cat. no. AF6088) and vascular cell adhesion molecule 1 (VCAM-1; 1:1,000; cat. no. DF6082) were purchased from Affinity Biosciences, Ltd. The hematoxylin staining kit (cat. no. AR1180-1) was obtained from Boster Biological Technology. Desktop centrifuge Neofuge 13 was a product of Heal Force. The ultra-high sensitivity chemiluminescence imaging system ChemiDocXRS ${ }^{+}$was from Bio-Rad Laboratories. The ultra-high resolution small animal color Doppler ultrasound and real-time imaging system Vevo ${ }^{\circledR} 2100$ was purchased from VisualSonics, Inc. The CX41 microscope was obtained from Olympus Corporation and the NovoCyte ${ }^{\mathrm{TM}}$ flow cytometer was obtained from Agilent Technologies, Inc.
$H / R$ cell model and drug treatments. HK-2 cells were suspended in culture medium and cultured overnight at $37^{\circ} \mathrm{C}$. The medium was refreshed every day until the cells grew to confluence. For H/R modeling, HK-2 cells were cultured to $80 \%$ confluence and hypoxia was induced by placing the cell culture in a hypoxic chamber with a $1 \% \mathrm{O}_{2}+93 \% \mathrm{~N}_{2}+5 \%$ $\mathrm{CO}_{2}$ gas mixture in DMEM without serum at $37^{\circ} \mathrm{C}$ for $24 \mathrm{~h}$. After the hypoxic treatment, the cells were reoxygenated in normal oxygen at $37^{\circ} \mathrm{C}$ for $3 \mathrm{~h}$ and collected for further analysis via centrifugation at $500 \mathrm{x}$ g for $10 \mathrm{~min}$ at room temperature. DEX $(0.01 \mathrm{nM})$ and 4-PBA $(5 \mathrm{mM})$ were added to the cells at $37^{\circ} \mathrm{C} 1 \mathrm{~h}$ prior to modeling. Untreated cells were used as the control.

Apoptosis analysis. Apoptosis was detected using flow cytometry using Annexin V-FITC/PI Apoptosis kit. A total of $1-3 \times 10^{6}$ cells were pelleted and washed twice with $1 \mathrm{ml}$ PBS, resuspended in $300 \mu \mathrm{l}$ pre-chilled binding buffer, and then $3 \mu \mathrm{l}$ annexin V-FITC and $5 \mu \mathrm{l}$ PI-PE were added to cells. After gentle mixing, the cells were incubated at room temperature in the dark for $10 \mathrm{~min}$, then loaded with $200 \mu \mathrm{l}$ precooled binding buffer into the cytometer for analysis according to the manufacturer's instructions (NovoCyte Flow Cytometer System; Agilent Technologies, Inc.) using NovoExpress (v.6.2; Agilent Technologies, Inc.).

Western blotting. Cells were lysed in lysis buffer on ice bath for $30 \mathrm{~min}$ and centrifuged at $4^{\circ} \mathrm{C}$ at $500 \mathrm{x}$ g for $10 \mathrm{~min}$. Proteins in the supernatant were quantified using a BCA kit according to the supplier's protocols. After denaturing, the proteins were separated on $12 \%$ gels using SDS-PAGE for 1-2 $\mathrm{h}$ and transferred to PVDF membranes for western blotting analysis. The membranes were blocked with $5 \%$ nonfat milk powder at room temperature for $2 \mathrm{~h}$ and then incubated with antibodies against eIF2 $\alpha$, CypD, cytochrome $c$, ICAM-1, VCAM-1 and GAPDH (used as internal reference) at $4^{\circ} \mathrm{C}$ overnight, followed by incubation and with the secondary goat horseradish peroxidase-conjugated antibodies for mouse and rabbit IgG at room temperature for 1-2 $\mathrm{h}$. After visualization using enhanced chemiluminescence solution (cat. no. 34077; Thermo Fisher Scientific, Inc.), immunoreactive bands were captured using the gel imaging system. The gray value of each band was analyzed by Quantity One software (version 4.6; Bio-Rad Laboratories, Inc.).

$H / R$ animal model and drug treatment. After fasting for 16-24 h, mice were anaesthetized using an intraperitoneal injection of $100 \mathrm{mg} / \mathrm{kg}$ ketamine (Jiangsu Hengrui Medicine Co., Ltd.) and $10 \mathrm{mg} / \mathrm{kg}$ xylazine (Hubei Xinmingtai Chemical Co., Ltd.). An incision along the abdominal midline was made to expose bilateral renal pedicles. For the sham group, the right kidney was removed, and the abdominal cavity was closed in $45 \mathrm{~min}$. For the IR model, the right kidney was removed, the left renal pedicle was clamped with a non-invasive artery clamp for $45 \mathrm{~min}$ and then the clamp was released to re-perfuse for $24 \mathrm{~h}$. For drug treatments, DEX $(10 \mu \mathrm{g} / \mathrm{kg})$ or $4-\mathrm{PBA}(400 \mathrm{mg} / \mathrm{kg})$ was administered by intraperitoneal injection $30 \mathrm{~min}$ before the artery clamping. If the color of the kidney turned from purple/black to red and the animal was awake with $3 \mathrm{~h}$ of 

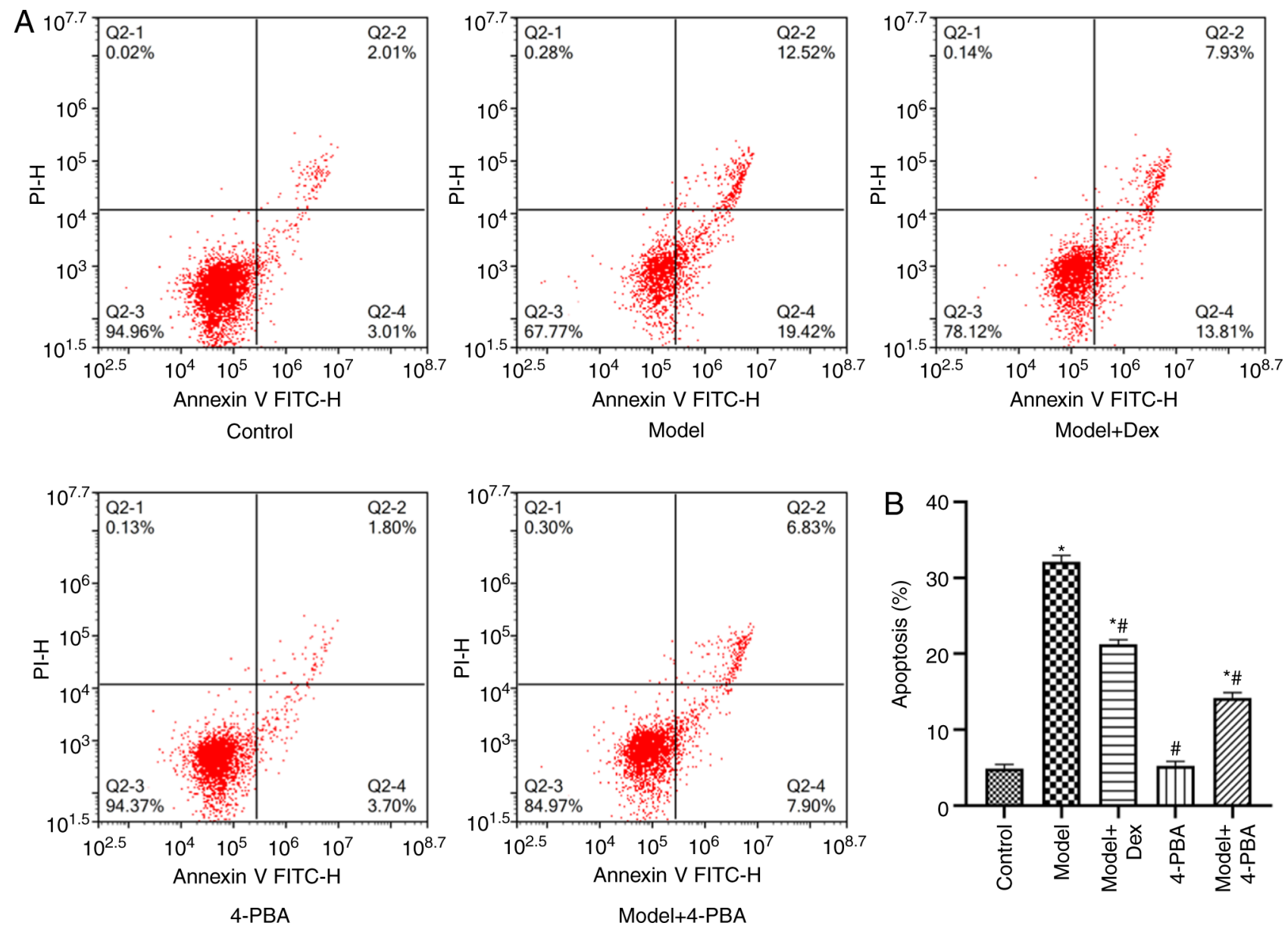

4-PBA

Figure 1. Flow cytometry analysis of apoptosis in HK-2 cells following hypoxia/reoxygenation and DEX and 4-PBA treatment. (A) Flow cytometry results and (B) quantified apoptotic rate. ${ }^{*} \mathrm{P}<0.05$ vs. control; ${ }^{\#} \mathrm{P}<0.05$ vs. model. DEX, dexmedetomidine; 4-PBA, 4-phenylbutyric acid.

surgery, the re-perfusion was considered successful. At $24 \mathrm{~h}$ after successful modeling, the renal arterial resistance index (RRI) was determined using high-resolution color Doppler ultrasound. After the measurement, mice were euthanized as aforementioned and kidney tissues were collected to measure the weight and size using an electronic balance and digital Vernier caliper.

Immunohistochemical assay. Tissues fixed in 4\% paraformaldehyde at $4^{\circ} \mathrm{C}$ for $12 \mathrm{~h}$ were embedded in paraffin, sectioned at $10 \mu \mathrm{m}$ thickness, were baked at $65^{\circ} \mathrm{C}$ for $2 \mathrm{~h}$, soaked in xylene for $10 \mathrm{~min}$ and rehydrated using a descending ethanol gradient series. After antigen retrieval with citrate buffer (cat. no. C1032; Beijing Solarbio Science \& Technology Co., Ltd.) at $100^{\circ} \mathrm{C}$ for $20 \mathrm{~min}$ and washing with PBS, the slices were-incubated with diluted antibodies against ICAM-1 and VCAM-1 overnight at $4^{\circ} \mathrm{C}$ and secondary antibody (anti-rabbit IgG labeled horseradish peroxidase) at room temperature for $10 \mathrm{~min}$. The diaminobenzidine and hematoxylin chromogen (Dako; Agilent Technologies, Inc.) were used to stain the slices at $25^{\circ} \mathrm{C}$ for $1 \mathrm{~h}$ to visualize immunoreactive bands. The sections were subsequently mounted with resin (cat. no. CW0136; CoWin Biosciences and examined under a light microscope and the intensity of staining was relatively qualified using ImageJ software (v1.4; National Institutes of Health) to calculate the positive area.
Statistical analysis. All data are expressed as the mean \pm standard error of the mean obtained from at least three independent experiments. Statistical comparisons between experimental and control groups were assessed using one-way ANOVA with Tukey's post hoc test. The data were analyzed by SPSS version 11.5 for Windows (SPSS Inc.). $\mathrm{P}<0.05$ was considered to indicate a statistically significant difference.

\section{Results}

DEX and 4-PBA decrease apoptosis. Flow cytometry studies revealed that compared with that in normal HK-2 cells, apoptosis was significantly increased in the model H/R HK-2 cells $(\mathrm{P}<0.05)$; furthermore, DEX significantly lowered apoptosis in the H/R HK-2 cells ( $\mathrm{P}<0.05$; Fig. 1A and B). 4-PBA did not significantly impact the apoptosis of $\mathrm{HK}-2$ cells, but significantly decreased the apoptosis of H/R HK-2 cells compared with the model only and control groups $(\mathrm{P}<0.05$; Fig. 1B). The apoptosis rate of the model + 4-PBA group was slightly but non-significantly $(\mathrm{P}>0.05)$ lower compared with that of model + DEX group, indicating that 4-PBA had a significant inhibitory effect on the apoptosis of HK-2 cells induced by hypoxia reoxygenation and the effect was equivalent to that of DEX. The data suggested that combined treatment of DEX and 4-PBA reduced apoptosis-induced by $\mathrm{H} / \mathrm{R}$ treatment, 


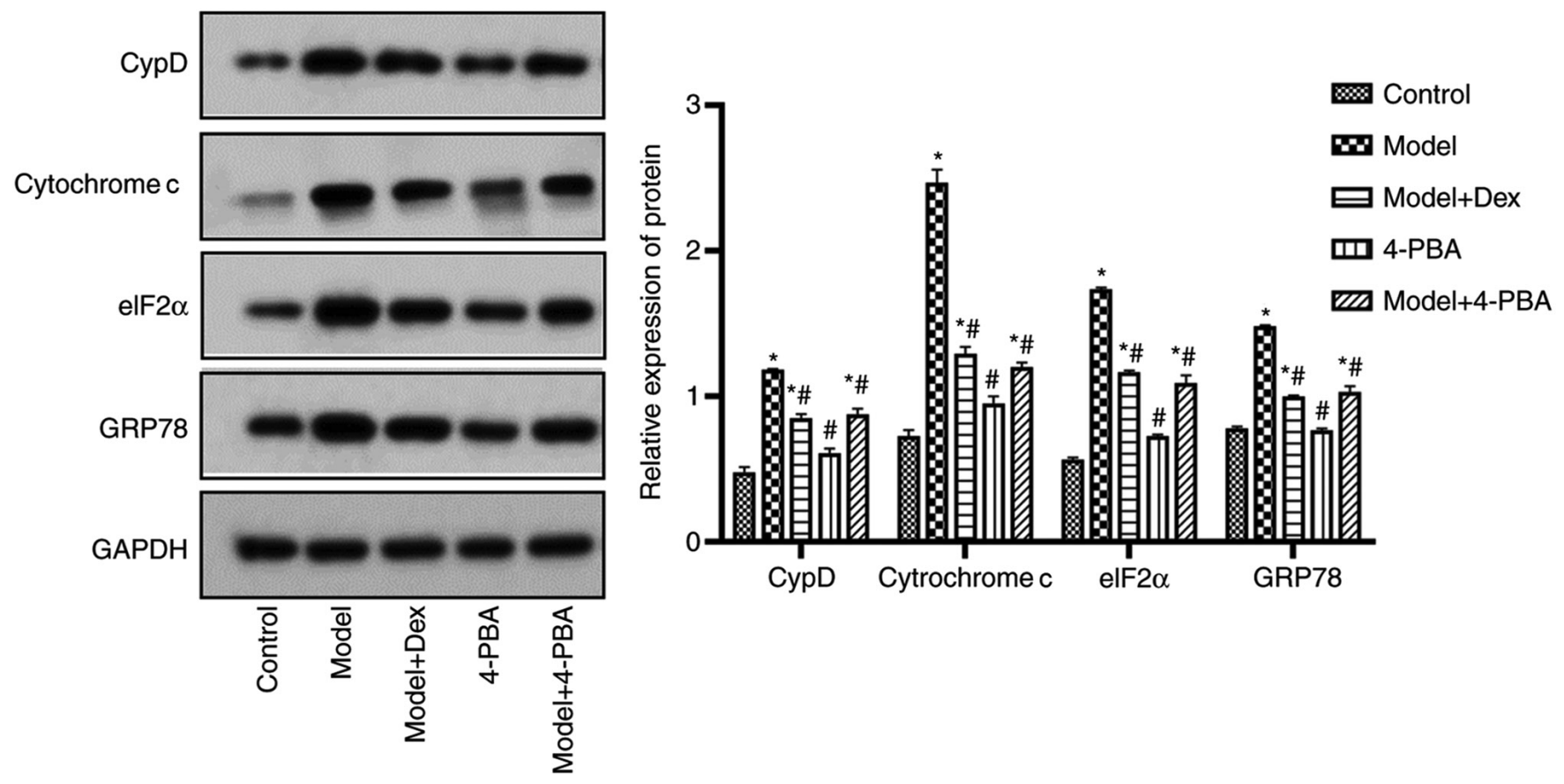

Figure 2. Expression levels of CypD, cytochrome $c$, eIF2 $\alpha$ and GRP78 in HK-2 cells following hypoxia/reoxygenation and DEX and 4-PBA treatment. The left panel presents representative western blotting, and the right panel shows the quantified relative protein levels. " $\mathrm{P}<0.05$ vs. control; ${ }^{~} \mathrm{P}<0.05$ vs. model. CypD, cyclophilin D; eIF2 $\alpha$, eukaryotic translation initiation factor $2 \alpha$; GRP78, glucose-regulated protein 78.

DEX and 4-PBA downregulate the expression levels of CypD, cytochrome $c$, eIF $2 \alpha$ and GRP78. Western blotting was performed to assess the impact of DEX and 4-PBA on CypD, cytochrome $c$, eIF $2 \alpha$ and GRP78 expression levels in H/R cells as presented in Fig. 2. The results indicated that 4-PBA did not significantly change the expression levels of these proteins compared with the control. However, all of these proteins were significantly upregulated after $\mathrm{H} / \mathrm{R}$ modeling ( $\mathrm{P}<0.05$; Fig. 2). DEX and 4-PBA significantly downregulated the expression levels of these proteins in H/R HK-2 cells compared with the model group $(\mathrm{P}<0.05$; Fig. 2). The data indicated that combined treatment of DEX and 4-PBA reduced the expression of CypD, cytochrome $c$, eIF2 $\alpha$ and GRP78 after H/R treatment.

$D E X$ and 4-PBA alleviate ischemia-reperfusion injury (IRI). At the time of measurement, the renal volume and weight were not different among all groups (Fig. 3). The RRI by color Doppler ultrasound indicated that RRI was significantly increased following ischemia-reperfusion modeling compared with that in the control $(\mathrm{P}<0.05)$. However, it was decreased significantly after DEX and 4-PBA treatment ( $\mathrm{P}<0.05$; Fig. 3$)$. Compared with that of the DEX-treated group, the RRI of the 4-PBA-treated group was similar and the difference between the groups was not significant $(\mathrm{P}>0.05)$, which indicated that the treatments produced similar effects.

DEX and 4-PBA downregulate the expression levels of ICAM-1 and vascular adhesion molecule (VCAM)-1. Western blotting and immunohistochemistry were used to examine the expression of ICAM-1 and VCAM-1 in the kidney tissues. Western blotting demonstrated that the expression levels of ICAM-1 and VCAM-1 were significantly increased in the model compared with those in the control, and that they were significantly decreased after DEX and 4-PBA treatment $(\mathrm{P}<0.05$; Fig. 4). The expression levels were also significantly different between model +4 -PBA and model + DEX groups in immunohistochemistry assessment of the two proteins $(\mathrm{P}<0.05$; Fig. 5). These findings indicated that ICAM-1 and VCAM-1 were downregulated following DEX and 4-PBA treatment, compared with the model group.

\section{Discussion}

At present, the main measures to decrease RIRI and protect renal function include renal hypothermia, a short surgical duration and selective blocking of renal artery branches (15). However, these measures are often insufficiently effective, particularly in cases with complex conditions, where it is not possible to avoid a long operation time and the irreversible damage caused by long-term blocking of renal artery branches (15). The manner in which to alleviate and decrease RIRI is still a clinical challenge. The purpose of the present study was to explore the effect of 4-PBA on RIRI and its possible mechanism. The results indicated that 4-PBA could significantly decrease H/R-induced apoptosis in HK-2 cells and decrease the renal artery blood flow resistance in RIRI mice. In addition, 4-PBA could significantly decrease the expression of ICAM-1 and VCAM-1 in the kidney of RIRI mice, leading to a significant alleviation of RIRI.

CypD is a component protein of the mitochondrial permeability transition pore (16). Mitochondria are the main sites to produce reactive oxygen species and also the target of oxidative damage. One of the mitochondrial responses to oxidative stress and thiols is the binding of adenonucleotide transferase and CypD (16). Studies have revealed that depletion of CypD in cardiomyocytes results in significantly less susceptibility to 

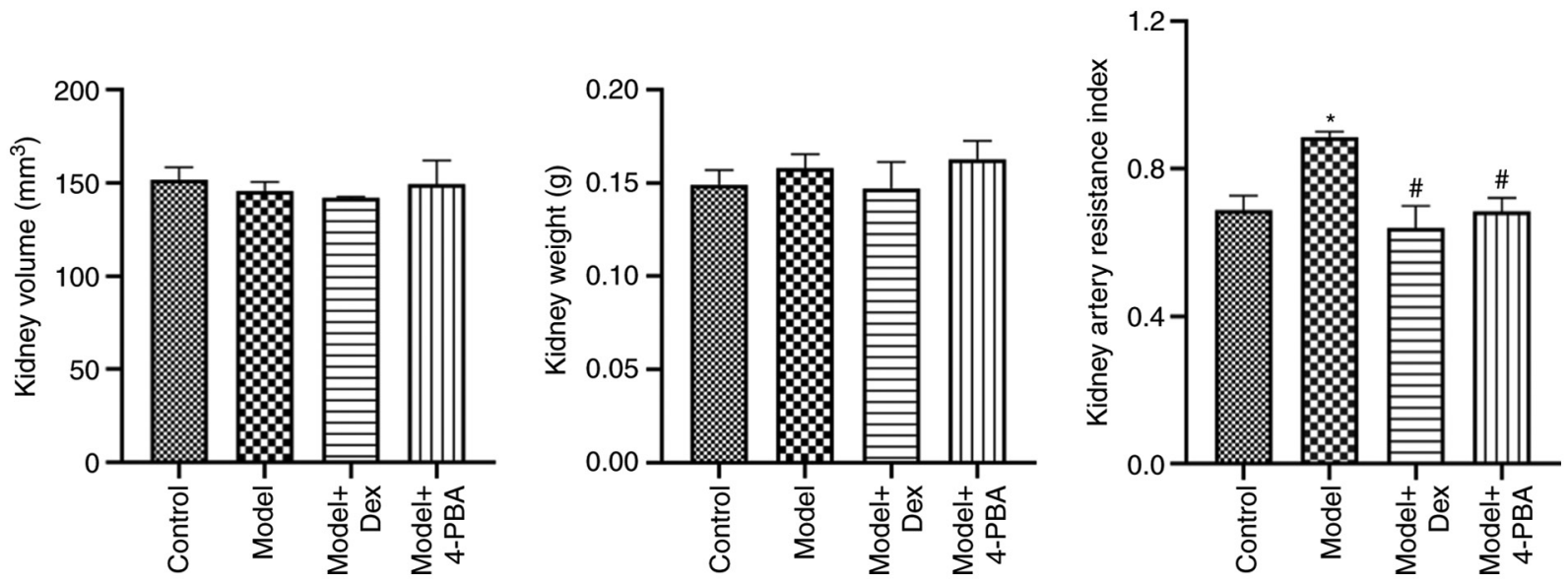

Figure 3. Characterization of kidney volume, weight and kidney artery resistance index of mice following ischemia-reperfusion and DEX and 4-PBA treatment. ${ }^{\mathrm{P}}<0.05$ vs. control; ${ }^{\mathrm{P}} \mathrm{P}<0.05$ vs. model. DEX, dexmedetomidine; 4-PBA, 4-phenylbutyric acid.
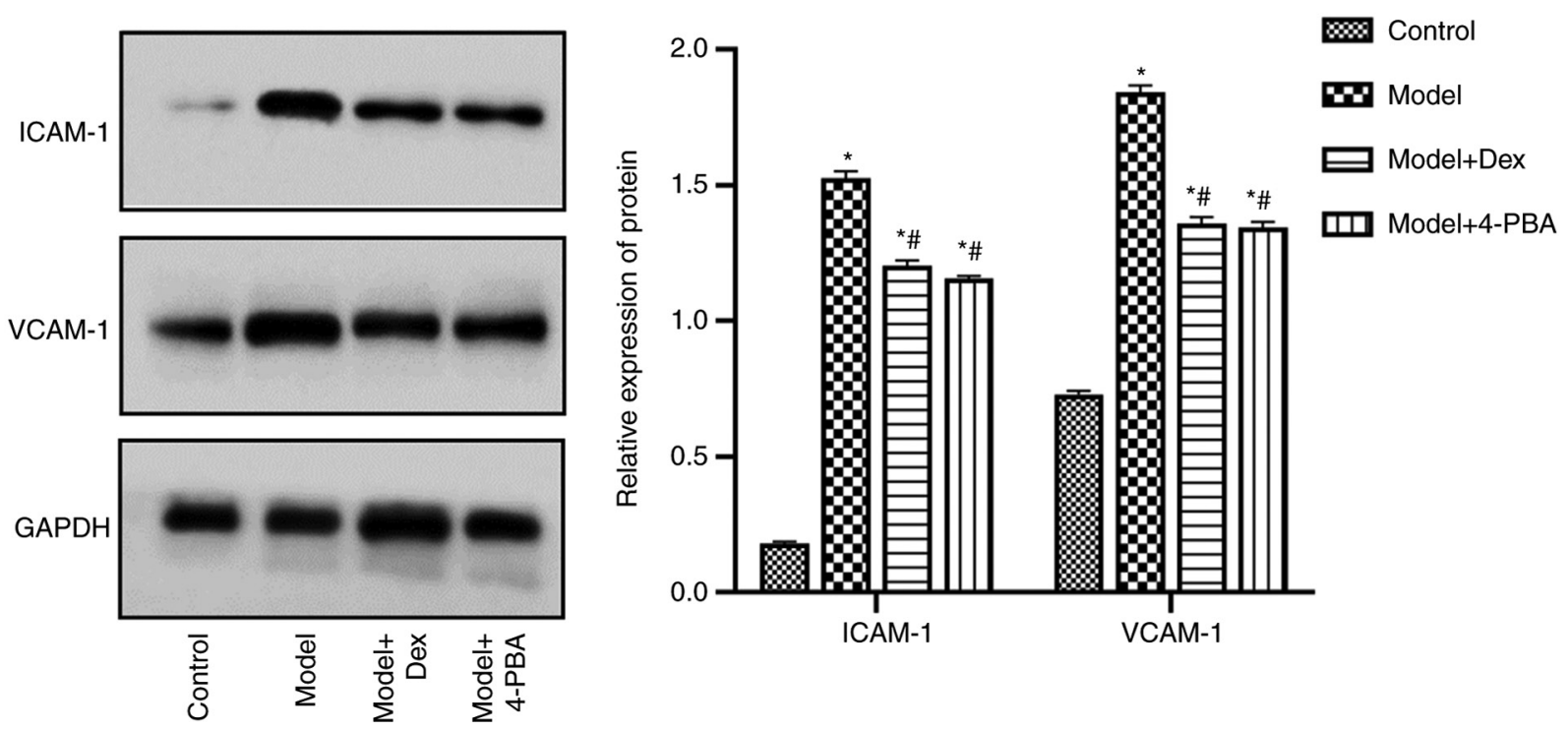

Figure 4. Western blotting analysis of the expression levels of ICAM-1 and VCAM-1 in mouse kidneys following ischemia-reperfusion, DEX and 4-PBA treatment. The right panel presents representative western blotting, and the left panel shows the relative expression levels. "P $<0.05$ vs. control; " $\mathrm{P}<0.05$ vs. model. DEX, dexmedetomidine; 4-PBA, 4-phenylbutyric acid; ICAM-1, intercellular adhesion molecule-1; VCAM-1, vascular adhesion molecule-1.

cell death induced by oxidative stress and calcium overload, and decreased synthesis of apoptosis-related proteins $(16,17)$. Cytochrome $c$ is a major component in the respiratory chain and plays a notable role in redox and energy metabolism. At the same time, cytochrome $c$ is the key trigger that initiates mitochondrial apoptosis (18). Cytochrome $c$ can activate downstream caspase 3 through a cascade reaction, leading to apoptosis (19). EIF2 $\alpha$ is a key regulatory protein in the process of translation initiation, which is largely dependent on its phosphorylation level (20). PERK is a transmembrane protein kinase in the endoplasmic reticulum (ER), which can phosphorylate eIF $2 \alpha$. Under ERS, phosphorylated PERK inactivates eIF $2 \alpha$ by phosphorylating the $\alpha$-subunit of eIF2, blocking the transformation from eIF2-GDP to eIF2-GTP (21). This affects the recruitment and initiation of initiator methionyl-transfer RNA and the 40S ribosomal subunit, resulting in the suspension of protein synthesis to decrease protein load in the ER and to restore cell homeostasis $(22,23)$. GRP78 is a notable molecular chaperone located in the ER, which plays a role in protein folding, transport and ERS response (24). As an ERS marker, GRP78 can bind to ERS-activated pro-apoptotic receptors to inhibit their signal transduction, thus protecting cells (25).

The present study revealed that 4-PBA could significantly decrease the expression of CypD, cytochrome $c$, eIF $2 \alpha$ and GRP78. In addition, H/R-induced apoptosis in HK-2 cells was decreased. We hypothesized that downregulation of these genes is likely due to 4-PBA suppressing the release of $\mathrm{H} / \mathrm{R}$-induced inflammatory factors. However, further studies are needed to elucidate the possible molecular mechanisms.

ICAM-1 and VCAM-1 are members of the immunoglobulin superfamily. Under the stimulation of inflammatory cytokines and bacterial endotoxins, they are expressed on the surface of activated endothelial cells to mediate the adhesion and exudation of leukocytes; they also have close relationship with the inflammatory mechanism of IRI $(26,27)$. The animal experiments of the present study demonstrated that 4-PBA could 


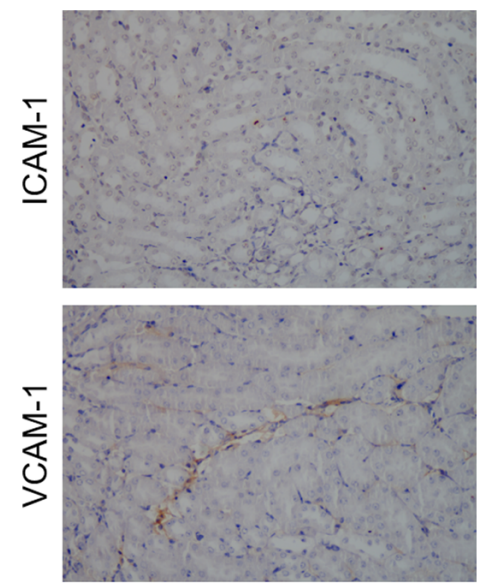

Control
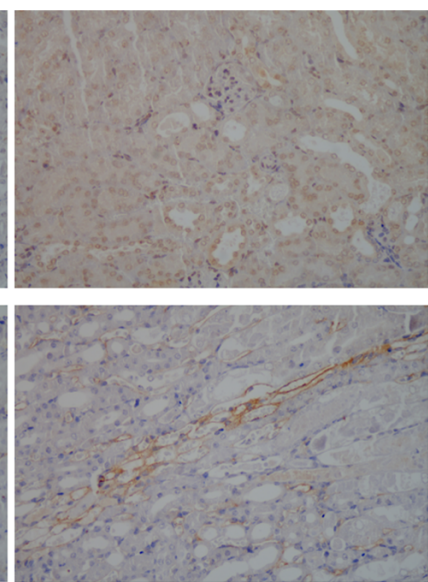

Model
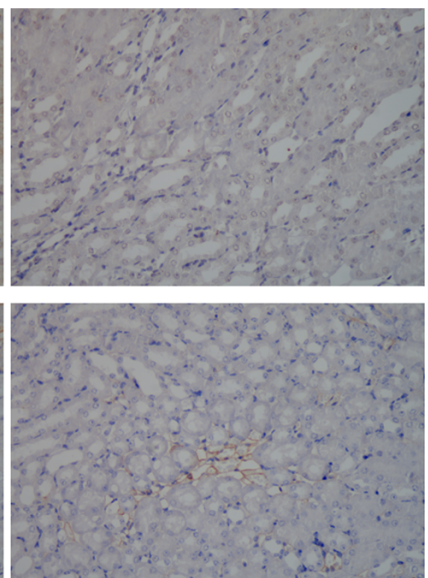

Model+Dex
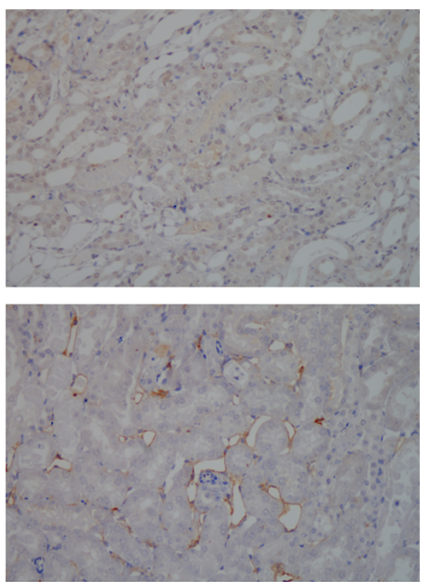

Model+4-PBA

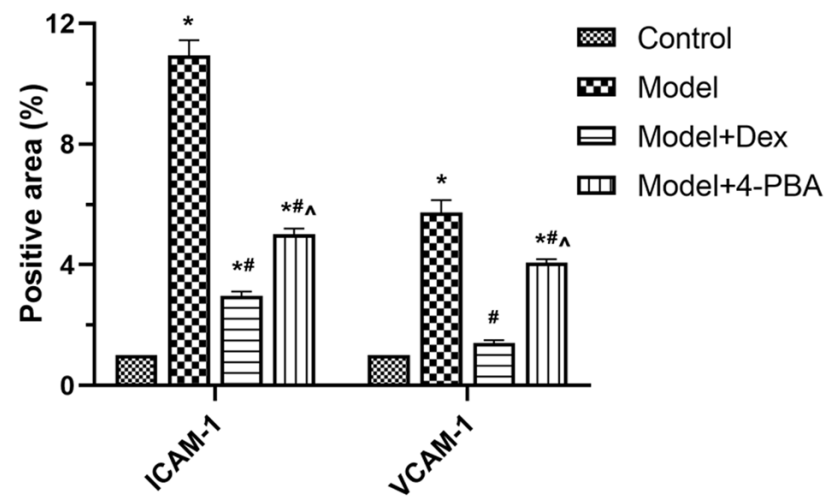

Figure 5. Immunohistochemistry assessment of the expression levels of ICAM-1 and VCAM-1 in mice following ischemia-reperfusion and DEX and 4-PBA treatment (magnification, $\mathrm{x} 400$ ). The upper panel presents immunohistochemistry results, and the lower panel shows the quantified relative expression levels. ${ }^{*} \mathrm{P}<0.05$ vs. control; ${ }^{\#} \mathrm{P}<0.05$ vs. model; ${ }^{\wedge} \mathrm{P}<0.05$ vs. model + DEX. DEX, dexmedetomidine; 4-PBA, 4-phenylbutyric acid; ICAM-1, intercellular adhesion molecule-1; VCAM-1, vascular adhesion molecule-1.

significantly decrease the expression of ICAM-1 and VCAM-1. In the IRI lesion, inflammatory cytokines are released locally, resulting in increased expression of ICAM-1 and VCAM-1, and increased cell adhesion. As a consequence, a large number of leukocytes adhere to vascular endothelial cells, resulting in obstruction of blood vessels and decreased blood flow $(26,27)$. Meanwhile, leukocytes outside blood vessels may produce free radicals, proteolytic enzymes and other toxic substances, causing vascular injury and bleeding $(28,29)$. Taken together, the evidence suggests that 4-PBA could downregulate the expression of CypD, cytochrome $c$, eIF2 $\alpha$ and GRP78 to decrease cellular oxygen free radicals, leading to a decrease in apoptosis and ERS. As a consequence, IRI and intercellular adhesion are decreased to healthy levels; moreover, the expression levels of ICAM-1 and VCAM-1 are reversed by downregulation of the expression of CypD, cytochrome $c$, eIF2 $\alpha$ and GRP78 proteins to healthy levels.

There are limitations to the present study. For example, the renal function of mice was not assessed using hematoxylin-eosin or Periodic acid-Schiff staining to detect pathological changes in the kidney, and the ERS was addressed using only two indicators, eIF2 $\alpha$ and GRP78. In further studies, more indicators and parameters, including pathological examination, should be applied for an improved understanding of changes in renal function following treatment.
In conclusion, to the best of our knowledge, the present study has demonstrated that 4-PBA can decrease RIRI in HK-2 cells and mice for the first time. The therapeutic effects were mediated via downregulation of CypD, cytochrome $c$, eIF2 $\alpha$, GRP78, ICAM-1 and VCAM-1, and were validated with human cell and animal studies.

\section{Acknowledgements}

Not applicable.

\section{Funding}

This study was supported by Jiangxi Provincial Department of Education (grant no. 180061).

\section{Availability of data and materials}

The datasets used and/or analyzed during the current study are available from the corresponding author on reasonable request.

\section{Authors' contributions}

XW and HG designed the study. XW, YZ and KW performed the experiments and the data analysis. XW and KW confirm 
the authenticity of all the raw data. XW, YZ and HG drafted the manuscript. All authors have read and approved the final manuscript.

\section{Ethics approval and consent to participate}

All animal experiments and animal care were conducted in accordance with the criteria of the Laboratory Animals Welfare Act and the Guide for the Care and Use of Laboratory Animals (30) provided by the Animal Research Ethics Committee of the First Affiliated Hospital of Nanchang University. Animal studies were approved by The Animal Research Ethics Committee of The First Affiliated Hospital of Nanchang University (approval no. 2019-067).

\section{Patient consent for publication}

Not applicable.

\section{Competing interests}

The authors declare that they have no competing interests.

\section{References}

1. Kusch A,Hoff U,Bubalo G,Zhu Y,Fechner M, Schmidt-Ullrich R, Marko L, Müller DN, Schmidt-Ott KM, Gürgen D, et al: Novel signalling mechanisms and targets in renal ischaemia and reperfusion injury. Acta Physiol (Oxf) 208: 25-40, 2013.

2. de Vries DK, Khairoun M, Lindeman JH, Bajema IM, de Heer E, Roest M, van Zonneveld AJ, van Kooten C, Rabelink TJ, Schaapherder AF and Reinders ME: Renal ischemia-reperfusion induces release of angiopoietin-2 from human grafts of living and deceased donors. Transplantation 96: 282-289, 2013.

3. Perico N, Cattaneo D, Sayegh MH and Remuzzi G: Delayed graft function in kidney transplantation. Lancet 364: 1814-1827, 2004.

4. Kalyanaraman B: Teaching the basics of redox biology to medical and graduate students: Oxidants, antioxidants and disease mechanisms. Redox Biol 1: 244-257, 2013.

5. Wang Y, He J, Pei X and Zhao W: Systematic review and meta-analysis of mesenchymal stem/stromal cells therapy for impaired renal function in small animal models. Nephrology (Carlton) 18: 201-208, 2013.

6. Song JY, Meng LQ and Li XM: Therapeutic application and prospect of Astragalus membranaceus and Angelica sinensis in treating renal microvascular lesions. Zhongguo Zhong Xi Yi Jie He Za Zhi 28: 859-861, 2008 (In Chinese).

7. Afonso J and Reis F: Dexmedetomidine: Current role in anesthesia and intensive care. Rev Bras Anestesiol 62: 118-133, 2012

8. Shukry M and Miller JA: Update on dexmedetomidine: Use in nonintubated patients requiring sedation for surgical procedures. Ther Clin Risk Manag 6: 111-121, 2010.

9. Patil RH, Naveen Kumar M, Kiran Kumar KM, Nagesh R, Kavya K, Babu RL, Ramesh GT and Chidananda Sharma S: Dexamethasone inhibits inflammatory response via down regulation of AP-1 transcription factor in human lung epithelial cells Gene 645: 85-94, 2018

10. Luo T, Chen B and Wang X: 4-PBA prevents pressure overload-induced myocardial hypertrophy and interstitial fibrosis by attenuating endoplasmic reticulum stress. Chem Biol Interact 242: 99-106, 2015.

11. Wang Z, Zheng S, Gu Y, Zhou L, Lin B and Liu W: 4-PBA enhances autophagy by inhibiting endoplasmic reticulum stress in recombinant human beta nerve growth factor-induced PC12 cells after mechanical injury via PI3K/AKT/mTOR signaling pathway. World Neurosurg 138: e659-e664, 2020.

12. Zeng M, Sang W, Chen S, Chen R, Zhang H, Xue F, Li Z, Liu Y, Gong Y, Zhang $\mathrm{H}$ and Kong X: 4-PBA inhibits LPS-induced inflammation through regulating ER stress and autophagy in acute lung injury models. Toxicol Lett 271: 26-37, 2017.
13. Bhardwaj R, Tandon C, Dhawan DK and Kaur T: Effect of endoplasmic reticulum stress inhibition on hyperoxaluria-induced oxidative stress: Influence on cellular ROS sources. World J Urol 35: 1955-1965, 2017.

14. Association AVM: AVMA guidelines for the euthanasia of animals. 2013

15. Ng CK, Gill IS, Patil MB, Hung AJ, Berger AK, de Castro Abreu AL, Nakamoto M, Eisenberg MS, Ukimura O, Thangathurai $\mathrm{D}$, et al: Anatomic renal artery branch microdissection to facilitate zero-ischemia partial nephrectomy. Eur Urol 61: 67-74, 2012.

16. Ye F, Li X, Liu Y, Jiang A, Li X, Yang L, Chang W, Yuan J and Chen J: CypD deficiency confers neuroprotection against mitochondrial abnormality caused by lead in SH-SY5Y cell. Toxicol Lett 323: 25-34, 2020.

17. Ramachandran A, Lebofsky M, Baines CP, Lemasters JJ and Jaeschke H: Cyclophilin D deficiency protects against acetaminophen-induced oxidant stress and liver injury. Free Radic Res 45: 156-164, 2011.

18. Liu K, Shu D, Song N, Gai Z, Yuan Y, Li J, Li M, Guo S, Peng J and Hong $\mathrm{H}$ : The role of cytochrome $\mathrm{c}$ on apoptosis induced by Anagrapha falcifera multiple nuclear polyhedrosis virus in insect Spodoptera litura cells. PLoS One 7: e40877, 2012.

19. McManus MJ and Franklin JL: Dissociation of JNK activation from elevated levels of reactive oxygen species, cytochrome c release, and cell death in NGF-Deprived sympathetic neurons. Mol Neurobiol 55: 382-389, 2018.

20. Palam LR, Baird TD and Wek RC: Phosphorylation of eIF2 facilitates ribosomal bypass of an inhibitory upstream ORF to enhance CHOP translation. J Biol Chem 286: 10939-10949, 2011.

21. Dias-Teixeira KL, Calegari-Silva TC, Medina JM, Vivarini ÁC, Cavalcanti Á, Teteo N, Santana AKM, Real F, Gomes CM, Pereira RMS, et al: Emerging role for the PERK/eIF2 $\alpha / A T F 4$ in human cutaneous leishmaniasis. Sci Rep 7: 17074, 2017.

22. Page AB, Owen CR, Kumar R, Miller JM, Rafols JA, White BC, DeGracia DJ and Krause GS: Persistent eIF2alpha(P) is colocalized with cytoplasmic cytochrome c in vulnerable hippocampal neurons after 4 hours of reperfusion following 10-minute complete brain ischemia. Acta Neuropathol 106: $8-16,2003$

23. Chesnokova E, Bal N and Kolosov P: Kinases of eIF2a switch translation of mRNA subset during neuronal plasticity. Int J Mol Sci 18: 2213, 2017.

24. Teng J, Liu M, Su Y, Li K, Sui N, Wang S, Li L, Sun Y and Wang Y: Down-regulation of GRP78 alleviates lipopolysaccharide-induced acute kidney injury. Int Urol Nephrol 50: 2099-2107, 2018.

25. Aksoy MO, Kim V, Cornwell WD, Rogers TJ, Kosmider B, Bahmed K, Barrero C, Merali S, Shetty N and Kelsen SG: Secretion of the endoplasmic reticulum stress protein, GRP78, into the BALF is increased in cigarette smokers. Respir Res 18: $78,2017$.

26. Small DL, Morley P and Buchan AM: Biology of ischemic cerebral cell death. Prog Cardiovasc Dis 42: 185-207, 1999.

27. Chaves KC, Peron JP, Chammas R, Turaça LT, Pesquero JB, Braga MS, Foguer K, Schor N and Bellini MH: Endostatin gene therapy stimulates upregulation of ICAM-1 and VCAM-1 in a metastatic renal cell carcinoma model. Cancer Gene Ther 19: 558-565, 2012.

28. Xing H, Sun S, Mei Y and Herman D: The protective effect of rosuvastatin on ischemic brain injury and its mechanism. J Huazhong Univ Sci Technolog Med Sci 26: 667-669, 2006.

29. Shen A, Yang J, Gu Y, Zhou D, Sun L, Qin Y, Chen J, Wang P, Xiao F, Zhang L and Cheng C: Lipopolysaccharide-evoked activation of $\mathrm{p} 38$ and JNK leads to an increase in ICAM-1 expression in Schwann cells of sciatic nerves. FEBS J 275: 4343-4353, 2008.

30. Cardon AD, Bailey MR and Bennett BT: The animal welfare Act: From enactment to enforcement. J Am Assoc Lab Anim Sci 51: 301-305, 2012.

This work is licensed under a Creative Commons Attribution-NonCommercial-NoDerivatives 4.0 International (CC BY-NC-ND 4.0) License. 